\title{
Workshops on Writing Science
}

\author{
Catalysed by Vigyan Prasar
}

18-30 September 2017 at IISER Thiruvananthapuram

2-14 October 2017 at IISER Pune

Eligibility: Post graduates in any branch of science, as well as to post graduates in Mass Communication/Journalism, aspiring to contribute scientific content to print media in India.

Minimum requirements for participation: Ability to understand and speak English, the language of the workshop. Demonstrable ability to write in English or in any other Indian language would be an advantage. The workshop in IISER TVM will have special preference to people interested in writing science in Tamil and Malayalam whereas the one in IISER Pune will entertain those who are interested in writing in Hindi and Marathi.

The workshop will have lectures, presentations, discussions, role-plays, demonstrations, hands-on practice, feedback, mentoring and highly interactive sessions. The focus will be on work done as individuals and in teams.

Maximum number of participants for the workshop is limited. The workshop is compulsorily residential. Boarding and lodging free for selected candidates. Re-imbursement of the cost of travel by 2 nd AC train fare, only for deserving candidates. No registration fee.

\section{Last date for receipt of application: 10th August 2017.}

Application form can be accessed at

https://goo.gl/forms/3cWvE4qKlq1yRsIv1

For more details see www.vigyanprasar.gov.in 